\title{
Familiarity and Its Impact on Consumer Decision Biases and Heuristics
}

\author{
C. WHAN PARK \\ V. PARKER LESSIG*
}

The impact of familiarity on consumer decision biases and heuristics is examined. Subjects at three different familiarity levels revealed interesting differences in perceptual category breadth, usage of functional and nonfunctional product dimensions, decision time, and confidence.

$\mathrm{T}$ wo major approaches are available for operationalizing and measuring product familiarity. One is to measure product familiarity in terms of how much a person knows about the product; the other is to measure familiarity in terms of how much a person thinks s/he knows about the product. According to the former, product familiarity may be examined with respect to the knowledge structure of an individual's long-term memory (LTM). According to the latter, product familiarity is based on the person's selfreport of how much s/he knows about the product (Lichtenstein and Fischhoff 1977). The former approach (amount of knowledge) contributes to understanding the impact of memory contents on the decision maker's evaluation and choice decisions; the latter (self-assessed familiarity) provides information about decision makers' (DM) systematic biases and heuristics in choice evaluations and decisions.

The objective of the present study is to examine, in a descriptive framework, decision (evaluation) biases and heuristics of consumers at different levels of familiarity, with specific attention to the impact on such informationprocessing heuristics as (1) perceptual category breadth, (2) use of functional and nonfunctional product dimensions, (3) decision time, and (4) confidence in choice.

\section{Construct of Product Familiarity}

The conceptualization of product familiarity in this study follows the DM's subjective familiarity assessment at three different levels. However, different individuals may em-

${ }^{*}$ C. Whan Park is Associate Professor, Graduate School of Business, University of Pittsburgh, Pittsburgh, PA 15260. V. Parker Lessig is Associate Dean and Professor, School of Business, University of Kansas, Lawrence, KS 66045 . The authors gratefully acknowledge the assistance of James R. Bettman. This research was supported by grants from the University of Kansas General Research Fund and from the University of Kansas School of Business Research Fund provided by the Fourth National Bank and Trust Company, Wichita. ploy different criteria in assessing familiarity, thus making comparisons across subjects difficult. This assessment is, therefore, made using a common base defined in terms of the subject's perceived knowledge of those dimensions important in the evaluation of the product.

Although later material will discuss in detail how the three levels of familiarity differ, to understand the ensuing theory section it would be helpful to be aware of the necessary conditions that were established for specifying a subject's level of familiarity with the product, a microwave oven. Specifically, in order to maximize the difference in self-assessed familiarity levels among subjects, the following three prior behavioral considerations were specified: (a) microwave oven information search experience; (b) microwave oven usage experience; and (c) microwave oven ownership status. A subject with no information-search experience, no product-usage experience, and nonownership was defined to have low familiarity (LF). A subject defined to have a moderate level of familiarity (MF) met conditions (a) and/or (b), but not (c). A subject classified as having high familiarity (HF) had search experience, usage experience, and was a microwave oven owner. For reasons to be discussed later, subjects in the MF and the HF groups were also provided with information (from Consumer Reports and Consumer Buying Guides) that would influence their self-assessed knowledge of the product, microwave ovens.

When familiarity is defined subjectively, controlling prior behavioral activities, it is treated as a "state" variable, and two points should be noted. First, the three familiarity groups are expected to differ in their information about the product, their subjective judgment of cue selection and processing (i.e., confidence in choosing and processing product attributes for a choice decision task), and their organization of product information in long-term memory. Second, due to differences in prior behavioral activities (prior interest in the product class), differences among the three groups may be expected in their motivational involvement with the experimental task. 


\section{THEORY}

\section{Perceptual Category Breadth}

In the present study, it is proposed that a decision maker's level of product familiarity affects her perceptual category breadth. According to Bruner (1957), perceptual category breadth refers to a DM's viewing the various levels of a given dimension, e.g., size, as belonging to different categories, e.g., small, medium, large. A dimension whose levels are assigned to few categories, i.e., where there is very little discrimination, is said to have broad category breadth, i.e., each category covers a large range of the dimension's levels (Bruner and Tajfel 1961; Pinson 1978).

Two factors are considered in examining category breadth-a cognitive capability and a psychological desire to differentiate among dimensional categories. In this study, category breadth assumes that the DM has both the cognitive ability to differentiate among dimensional categories and utility differences across these categories. A DM at LF (low familiarity) is expected to exhibit broad category breadth with respect to the functional product dimensions evaluated. The greater the number of categories created for the classification of a dimension's levels, i.e., the narrower the category breadth, the more difficult is the assignment of utility, because more classes exist that require evaluation. With only indirect information or experience (based on other product usage) to draw upon and with the absence of a well-established dimensional salience hierarchy, the assignment of utility to a large number of categories would be a difficult and frustrating task. One way of reducing frustration and cognitive burden would be to broaden a dimension's categories, i.e., reduce the number of perceptual categories on that dimension.

The DM at MF (moderate familiarity) has relevant and needed product information from previous usage experience and/or from information material provided to them. However, this DM has rather fragmented information about the meaning of dimensions and their salience hierarchy. Although this information is less than desired, it does aid in the DM's constructive process of a choice decision, thus reducing the degree of complexity involved. The MF DM is, therefore, capable of processing greater amounts of, and more complex, information (Park and Lessig 1977). To facilitate the development of this complex processing, the MF DM is expected to have narrower category breadth, i.e., more categories.

The HF DM is also expected to have a narrower category breadth than the DM at LF. Based on previous purchase decision making and on usage experience, the HF DM has developed a relatively complex and rigidly fixed utility for different levels of the various dimensions and their associated salience. This information, which is contained in long-term memory, is well defined and effectively retrieved for a specific choice decision task (Bettman 1979). Category breadth for the HF DM is not expected to differ from that of the MF DM. Although the HF DM has more experience and knowledge than the DM at MF and presum- ably is more capable of elaborate processing, she may employ categories less refined than those for which she is capable.

\section{Reliance on Price and Brand Name Information}

It is proposed that confidence in the utilization of brand name and price information is a function of the DM's familiarity level. Based upon previous discussion, it would seem that a DM at LF would find it less difficult and be more confident to extrapolate a product's utility from familiar concepts, such as the nonfunctional dimensions of price and brand name, than from unfamiliar product functional dimensions. This is consistent with Tversky and Kahneman's (1974) availability heuristic, which refers to the ease with which relevant instances of an event can be brought to mind.

The DM at HF is also expected to have high confidence in usage of brand name and price in the choice decision. Due to her previous purchase experience and knowledge about the brand that she owns, brand name and its price are expected to be placed very high on a dimensional salience hierarchy with high confidence. Due to her knowledge, the $\mathrm{DM}$ at $\mathrm{MF}$ is not expected to find it as difficult or as ambiguous to rely on the functional attributes as is the LF DM. Nor is the MF DM expected to have biases toward these dimensions that are as strong as those expressed by the HF DM. Given this and her ability to form utility through information on functional dimensions, the DM at MF would not feel as confident as LF and HF decision makers in evaluating choice options through the use of brand name and price information. The DM at MF is thus expected to have a higher confidence in functional dimensions than in price and brand-name dimensions.

\section{Decision Time}

The time required to make a decision is an important process-tracing measure (Hansen 1972). A number of investigators have described the consumer's decision task as consisting of two general stages (Howard and Sheth 1969; Park 1978; Payne 1976). In the first stage (choice reduction), the consumer reduces the number of available alternatives to a smaller set consisting of those options considered to be acceptable. In the second stage (choice selection), the consumer decides which alternative among those in the acceptable set is best. In the present study, decision time is hypothesized to be a function of both the level of the consumer's product familiarity and the stage of the decision process. Specifically, in the choice reduction state, the DM at MF is expected to require more time than either LF or HF decision makers.

This expectation is in line with Pollay's (1970) and Kiesler's (1966) findings on the nonmonotonic relationship between decision time and the difficulty of the choice task. According to Pollay and Kiesler, decision time is expected to increase as difficulty increases until the choice becomes quite difficult, at which point decision time decreases. On 
the other hand, the HF DM can achieve information search and processing efficiency due to her high level of familiarity (Sheth and Venkatesan 1968). This should result in the HF $\mathrm{DM}$ requiring less decision time than the DM at MF.

Decision time at the choice selection stage is expected to exhibit a different pattern due to changed perception of the task's difficulty. Specifically, during the choice reduction stage, the LF DM greatly simplified her choice task. This simplification now makes choice less difficult than at the reduction stage. The DM now feels motivated to select the brand that will give her the greatest utility. This motivation is expected to lead her to a careful and perhaps timeconsuming examination of additional information, due to lack of familiarity. Furthermore, when the remaining alternatives are barely discriminable on those dimensions upon which she heavily relied in the choice reduction state, the DM may be forced to consider additional information for which she is not an efficient information processor. On the other hand, the thoroughness of the choice reduction activities of the MF DM is likely to make her task simpler at the choice selection stage, although she is a careful information processor. This implies that the decision-making time difference between a DM at LF and a DM at MF would not be significant at the choice selection stage.

The HF DM is very efficient in processing information due to her well-established cognitive structure relating to decision alternatives. Therefore, she is expected to need a shorter decision time in her selection than is required by $\mathrm{LF}$ and MF decision makers.

\section{Choice Confidence}

The degree of confidence that the DM places on the appropriateness of the ultimate choice is viewed as being related to the DM's familiarity. ${ }^{1}$ The LF DM is expected to feel less confident than the MF DM, who has relevant knowledge for the decision-making task. The HF DM is also expected to have higher confidence in the choice than the MF DM, due to the HF DM's greater product usage experience and ownership. The confidence of the DM in her choice is thus hypothesized to increase monotonically with the level of her familiarity, for both the choice reduction stage and the choice selection stage.

\section{HYPOTHESES}

Based on the previous section, the following hypotheses are proposed:

H1: The perceptual category breadth on functional dimensions of the DM at a low level of familiarity is broader than that of DMs at either a moderate or a high level of familiarity.

\footnotetext{
'The confidence referred to in this section deals with the DM's overall confidence in the chosen brand. Confidence in the earlier setting referred to the DM's certainty that reliance on a particular product dimension will enhance her overall satisfaction. Similar distinctions have been made in previous research (Bennett and Harrell 1975).
}

H2: There is a significant difference between a DM at a moderate level of familiarity and DMs at either a low or a high familiarity level with respect to the processing and utilization of the nonfunctional dimensions of brand name and price. Specifically:

H2a: A DM at a moderate level of familiarity feels less confident in relying on price and brand name than DMs at either a low or a high level of familiarity.

H2b: A DM at a low level of familiarity feels more confident in relying on the nonfunctional dimensions of price and brand name than in relying on functional dimensions.

H2c: A DM at a moderate level of familiarity feels less confident in relying on the nonfunctional dimensions of price and brand name than in relying on functional dimensions.

H2d: A DM at a high level of familiarity feels as confident in relying on the nonfunctional dimensions of price and brand name as in relying on functional dimensions.

H3a: In reducing the alternatives to a smaller set of acceptable options, the decision time of a DM at a moderate level of familiarity is greater than that of DMs at either low or high levels of familiarity.

H3b: In selecting the best alternative in the acceptable set, the decision time of DMs at either low or moderate levels of familiarity is greater than that of a DM at a high level of familiarity.

H4: The DM at low familiarity feels less confident than moderate familiarity DMs, and the DM at a moderate familiarity level feels less confident than the DM at high familiarity. This holds for both the choice reduction and the choice selection stages.

\section{SUBJECTS}

Data were obtained from 99 women living in a midwestern college community. ${ }^{2}$ Each subject was contacted by telephone and asked whether she had ever searched for information about microwave ovens, had ever used a microwave oven, and currently owned a microwave oven. The responses were used to assign the subject to one of three familiarity groups: low familiarity (LF), 37 subjects; moderate familiarity (MF), 29 subjects; and high familiarity

\footnotetext{
${ }^{2}$ Eighty percent of the participants were randomly selected. The remaining 20 percent, although still representing a cross section of the community, were selected through convenience, in an attempt to balance the number of subjects possessing different degrees of product familiarity.
} 
(HF), 33 subjects. These three conditions were chosen to maximally discriminate among familiarity groups. Due to the absence of information search, product usage, and product ownership, the product familiarity and knowledge of subjects in the LF group can clearly be differentiated from those in the MF and HF groups. The MF subject, who had either search or usage experience (or both), but did not own the product, was not expected to have as much knowledge about dimensional importance as an individual who had prior purchasing experience and was a product user.

All of the MF subjects had prior product usage experience, but very few had searched for information regarding product attributes or differences among various brands. ${ }^{3}$ It was, therefore, believed desirable to provide the MF subjects with relevant information that could be used to further extend the subjective assessment of familiarity between the LF and MF groups. Furthermore, it was believed necessary to provide the HF subjects with the same information in order to create among the MF and HF subjects common definitional characterizations of the various microwave oven attributes. Thus, possible dependent variable differences between the MF and HF subjects would not be attributed to the information provided to the MF subjects.

The information given to the MF and HF subjects contained answers to questions commonly asked about microwave ovens (e.g., What are microwaves? Why do foods cook so quickly?) and descriptions of the functional nature of microwave oven attributes (e.g., What is an expanded scale timer? What is a safety start?). No information concerning price or brand name was given. The material was obtained from Consumer Reports, Consumer Buying Guide, and literature published by manufacturers to increase the study's reality, as functional attribute information of this type is available to the consumer. ${ }^{4}$ The MF and HF subjects were asked to examine this material prior to the personal interview and again prior to the experiment.

\footnotetext{
${ }^{3}$ When the MF group was near its target size (approximately 90 percent of the desired cell size), only two of these subjects had searched for information regarding differences among microwave oven brands or for information on product attribute performance. For the remaining MF subjects, selected through a convenience method, the authors did not identify how many had information search experience.

${ }^{4} T$ The information on the functional aspects of each microwave oven attribute was descriptive and provided both positive and negative information. For example, the following information was provided on the attribute "microwave leakage": "Microwave radiation is not the same as $\mathrm{X}$-ray or nuclear radiation. $\mathrm{X}$-rays and nuclear radiation can create electrically charged (that is, ionized) molecules capable of damaging cells by interfering with the process of cell division. In contrast, microwaves are nonionizing; any effect they may have is said to be thermal-the effect of heat on body tissue. The U.S. Bureau of Radiological Health has set limits to prevent harmful levels of microwave exposure. The emission standard for microwave ovens before sale sets a limit of one milliwatt of leakage per square centimeter $\left(1 \mathrm{~mW} / \mathrm{cm}^{2}\right)$. After sale, a maximum of 5 $\mathrm{mW} / \mathrm{cm}^{2}$ is permitted to allow for some deterioration of the oven over its lifetime."
}

\section{EXPERIMENTAL TASK AND DATA}

At the beginning of the interview, the subject indicated, through a five-point scale (ranging from "very familiar" coded as 5, to "unfamiliar" coded as 1 ), her opinion on which microwave oven features would be important in making a choice. ${ }^{5}$ The subject was then presented a matrix that described 15 models of microwave ovens in terms of ten dimensions: brand, price, type of microwave distribution, number of cooking levels, expanded scale timer, temperature setting, browner, microwave leakage, safety start, and usable oven capacity. Most of the descriptions accurately portrayed models available at the time. However, in a few instances the description was altered to avoid such problems as dominance, and to assure that a subject could be attracted to any given model depending on her choice criteria.

The subject examined the product description matrix and identified the options acceptable to her. She then indicated on a seven-point scale (extremely difficult to extremely easy) the difficulty of this choice reduction task. She also indicated, on a five-point scale (extremely confident to not confident at all), her confidence in the selections made with respect to her perceived certainty that the alternative(s) chosen will enhance her overall satisfaction. The options just identified as being acceptable were then reevaluated to determine which would be her first choice. The perceived difficulty of this choice selection task was also measured. The subject verbalized her thoughts (which were tape recorded) while performing both the choice reduction and the choice selection tasks; the time (rounded to the nearest minute) required to complete each task was also noted.

Also, for each of the ten microwave oven dimensions, the subject indicated on a seven-point scale her confidence that reliance on the dimension would enhance the satisfaction that she received from the oven chosen. Finally, for each dimension, she indicated (using an 11-point scale) the satisfaction she would receive from each of the dimension's levels, e.g., for the dimension "browner," the satisfaction associated with having and with not having a browner.

\section{FINDINGS}

\section{Verbal Protocol Examination}

Sixty-two of the tape-recorded protocols were transcribed and divided into coded phrases; ${ }^{6}$ these 62 contained 24,15 ,

\footnotetext{
${ }^{5}$ As a check on the previously determined familiarity levels, the responses were compared across the familiarity groups through one-way ANOVA using Duncan's Multiple Range test. The average familiarities across groups were significantly different at the 0.05 level and in the anticipated direction. The mean perceived familiarity for the LF, MF, and HF subjects were $1.89,3.13$, and 4.05 , respectively.

${ }^{6}$ Twenty of the 99 protocols were deleted because verbalizations could not be understood or because decisions were made without verbalizing their thoughts. Much to our distress, an additional 17 protocols were accidentally destroyed.
} 
and 23 subjects from the LF, MF, and HF groups, respectively. Two coders then independently examined each protocol statement to determine which product dimensions the subject had used in her choice task. For most of the 62 protocols, the two coders agreed in their assessment. Where there was disagreement, the subject had recognized variation across models on the dimension, but did not use the dimension in making a choice; such a dimension was discarded. The coders decided that the attribute was used for processing when examined in any of the following ways: attribute comparison process (e.g., attribute evaluation across brands), within-brand process (e.g., examining the attributes of a particular brand), and use of prior knowledge for evaluating attributes. ${ }^{7}$ Based on this understanding, the two coders agreed in their evaluation of all 62 protocols. Next, a third coder independently examined ten randomly selected protocols; his evaluations agreed with those of the other two. ${ }^{8}$

\section{Perceptual Category Breadth}

The subject's satisfaction associated with each of the dimension's levels was used to measure perceptual category breadth - the number of different satisfaction scale values that the subject assigned to the dimension's levels. The fewer the number of satisfaction categories used, the broader the breadth (Clayton and Jackson 1961). As the eight functional dimensions examined in this study differ in number of levels, the measure of breadth was standardized by dividing the number of categories assigned to a dimension by the number of levels of that dimension.

In analyzing perceptual category breadth, only the functional dimensions identified as having been processed and utilized by the subject in her choice task were considered; these dimensions were identified from the protocol data. A subject's standardized breadth scores on these dimensions were then averaged to measure her total category breadth. Using ANOVA, these scores (for the 62 protocol subjects) were then compared across the three familiarity groups. The mean values for the LF, MF, and HF groups were $0.66,0.76$, and 0.76 , respectively (the smaller the value, the broader the breadth). As expected (Hypothesis 1), a significant difference does not exist between the MF and HF groups. It should also be noted that although not significant $(p<0.16)$, the directional differences between LF category breadth and that of MF and $\mathrm{HF}$ are as expected.

\section{Reliance on Price and Brand Name}

Hypotheses 2 through $2 \mathrm{~d}$ are concerned with the difference of confidence in price and brand name among the three

\footnotetext{
${ }^{7}$ For more specific information regarding specific subcategories on each of these three general categories, see Bettman and Park (1980).

${ }^{8}$ Unless otherwise indicated, the analyses reported are based on the entire sample of 99 subjects. Although not reported, the same analyses were performed on the reduced sample of 62 subjects, with identical findings.
}

familiarity groups. These hypotheses were tested using responses to how confident a subject was in relying on the particular dimension in maximizing her overall satisfaction. Significant differences $(p<0.05)$ are noted when Duncan's multiple range test is used to compare across familiarity groups, confidence in price, and confidence in brand name. For brand the average confidence scores were 5.38, 4.79, and 5.85 for the LF, MF, and HF groups, respectively (7 $=$ very confident, $1=$ not confident at all). The $\mathrm{LF}, \mathrm{MF}$, and $\mathrm{HF}$ group averages for price were $5.19,5.10$, and 5.89 , respectively. The DM at MF shows less confidence than DMs at either low or high familiarity levels in relying on brand-name information. This observation is consistent with Hypothesis 2a. Also, as expected, when confidence in the usage of price information is examined, the DM at MF shows significantly less confidence than the DM at HF $(p<0.05)$; on the other hand, no significant difference is noted between the MF and the LF decision makers. These observations are inconsistent with Hypothesis 2a. Perhaps the LF subjects do not perceive price as being as useful an index of quality as is brand name (Park and Winter 1979).

An examination of the confidence scores for brand name and for price, as compared to those for the functional dimensions, reveals that the LF DM places higher confidence on the nonfunctional dimensions (5.36 average score for brand name and price) than on the functional dimensions (4.59 average for the eight functional dimensions). This contrast is statistically significant $(p<0.003)$ and supports Hypothesis $2 \mathrm{~b}$. On the other hand, the MF DM placed less confidence in her usage of price and brand name (mean score of 4.87) than on her usage of any of the functional dimensions (average score is 5.43); this difference is also significant $(p<0.02)$ and supports Hypothesis $2 c$. Finally, the HF DM appears to place as much confidence on price and brand name (average of 5.86) as on usage of the functional dimensions (average of 5.88).

Examination of Hypotheses 2b, 2c, and 2d reveals a significant interaction $(p<0.008)$ between level of familiarity and dimension type (but this does not assume independence for the within-subject responses).

\section{Decision Time}

Hypothesis 3a states that in the choice-reduction decision stage, the MF DM would require a significantly longer time to complete the task than DMs at either LF or HF. An ANOVA on the decision times for subjects in the three familiarity groups supports this hypothesis at the 0.01 level; the mean decision times were $6.70,9.38$, and 7.72 for subjects in the LF, MF, and HF groups, respectively.

Hypothesis $3 b$, on the other hand, states that LF and MF decision makers will require significantly more time than the HF DM in the choice selection decision stage. To test Hypothesis $3 \mathrm{~b}$, an adjustment in the data base was made to nullify (or standardize) the effect that differing numbers of choice options would have on decision time comparisons 
across familiarity groups. ${ }^{9}$ As a result of this standardization, when ANOVA (Duncan's Multiple Range test) is used to compare the subsets on the basis of decision time, differences in decision time are a reflection of familiarity and not the number of options evaluated. The mean decision time for the LF, MF, and HF subsets were 2.43, 2.14, and 1.04 , respectively $(p<0.08)$. Duncan's Multiple Range test showed the differences between the low and the high familiarity subsets and between the moderate and the high familiarity subsets to be marginally significant $(p<0.10)$. No significant difference was found between the low and the moderate familiarity subsets. These findings marginally support Hypothesis $3 \mathrm{~b}$.

These findings are further supported by an examination of the perceived difficulty of the decision task. ANOVA across the three familiarity groups on the perceived difficulty of the decision task at the choice reduction stage showed that the LF DM perceived the task to be more difficult than did MF and HF decision makers $(p<0.002)$. Because of her perceived task difficulty, the LF DM is expected to greatly simplify her decision task, reducing the decision time from that which would be expected from looking at perceived task difficulty only.

However, ANOVA showed no significant difference among the three groups in the level of task difficulty.

\section{Confidence in Decision}

Hypothesis 4 states that a DM's confidence in her choice decision increases monotonically with her level of familiarity in each of the two choice tasks. Subject responses on confidence in the decision were analyzed through Duncan's Multiple Range test; the findings partially support the hypothesis.

Specifically, confidence increases monotonically with the level of familiarity. At the choice reduction stage, the monotonic increases in confidence between the LF and the MF groups and between the LF and the HF groups are both significant at the 0.05 level; no significant difference was noted between the MF and the HF groups. The monotonic increases in confidence with familiarity is also noted at the choice selection stage with the difference between the low and the high familiarity groups also significant at the 0.05 level. No significant difference, however, is noted between

\footnotetext{
${ }^{9}$ This standardization was accomplished by comparing decision time (at the choice-selection stage) across three subsets, one for each of the three familiarity groups. Each subset contained 21 subjects. The subsets were constructed such that the number of subjects who evaluated " $x$ " choice options was the same for each of the subsets. Assume, for example, that four LF DMs, six MF DMs, and seven HF DMs evaluated three microwave ovens at the choice-selection stage; the LF, MF, and HF decision time subsets, then, would respectively contain all four of these LF DMs, four of the six MF DMs (selected randomly), and four of the seven HF DMs. Through such a procedure, the composition of the three subsets were identical in terms of the number of subjects who evaluated a given number of choice options. Equalizing across familiarity groups, the number of subjects evaluating " $x$ " choice options resulted in the exclusion of 36 of the 99 subjects from the standardized subsets.
}

the low and the moderate familiarity groups. This suggests that the LF group is perhaps more confident than they should be, and the MF group is less confident than they should be.

Unlike subjects in other familiarity groups, the LF sub. jects expressed greater confidence in their choice reduction decision than in their decision at the choice selection stage. Although this difference is not significant at the 0.05 level, it seems to support the implications of previous discussion on the LF DM's choice processes at the two different choice tasks, i.e., more elaborate processing at the choice selection stage led the LF group to think that they chose the right brand no matter how low their perceived level of knowledge.

\section{DISCUSSION}

The decision biases and heuristics revealed by the three groups of subjects may be close approximations of those used by consumers at different stages of familiarity. Specifically, the decision (evaluation) biases and heuristics revealed by the LF subjects are expected to be found among consumers who do not have prior product-usage experience, are not equipped with relevant information about brand differences and the functional product attributes (no information search), and do not own the product. The MF subjects' evaluation biases and heuristics may be representative of those who have some product-usage experience, possess relevant information, but do not currently own the product. The HF subjects' evaluation and decision biases are expected to be found among consumers who currently own the product and have relevant product knowledge.

These generalizations should, however, be made with some caution. Specifically, the present study and the study by Bettman and Park (1980), using the same design and subjects, were not able to address motivational differences among the three groups of subjects in terms of the degree of their prior interest in microwaves and the impact of this interest on the study's findings. These questions are important in view of the recent findings about the state-ofmind effect on utility formation and choice decision (Wright and Kriewall 1980).

It is highly likely that differences among the three groups exist in the subjects' enduring involvement (Rothschild and Houston 1980) or prior interest in the product. The LF subjects would have a lower prior interest in the product than the MF group who had some prior product-usage experience and relevant product-attribute information; the MF interest may have been heightened through receiving relevant information material. Similarly, the MF subjects would have a lower interest in the product than HF subjects who have actually purchased the product.

Did differences in enduring involvement (prior interest) affect the situational involvement (involvement with the experimental task)? A concern of particular interest could relate to the LF subjects. Could their low interest in the experimental task have affected the results (i.e., "I am not 
interested in microwave ovens, thus I'm not really interested in this 'choose a microwave oven' task."). Several of the study's findings do not appear to support this possible bias. If the LF subjects had little involvement in the experimental task, they might simply have given up, using significantly fewer attributes and requiring a substantially shorter decision time than subjects in the MF and HF groups. However, comparison of the number of dimensions used in the choice-reduction stage revealed no significant difference among the three groups; the average number of dimensions used by the LF, MF, and HF subjects were $5.58,6.60$, and 6.04 , respectively. Similarly, at the choiceselection stage, no significant difference among the groups was noted; at this stage the average number of dimensions used were $2.71,2.58$, and 2.68 for the $\mathrm{LF}, \mathrm{MF}$, and $\mathrm{HF}$ groups, respectively. Also, careful examination of the protocols revealed that most of the subjects in the LF groups tried to infer the meaning of various product attributes based on experience with other products, and lamented their lack of knowledge about microwave ovens (Bettman and Park 1980).

Decision-time comparisons among the three groups also do not appear to support the notion of a bias among the LF subjects. Although there was a significant difference in decision time at the choice-reduction stage between the LF and MF subjects, and between the MF and HF subjects, there was no significant difference between subjects in the LF and the HF groups. Furthermore, at the choice-selection stage, the LF group required the longest time to make a decision, whereas the HF subjects required the least. If the level of prior interest had influenced the subjects' decision during the experimental task, the reverse decision time order would have been expected. It should also be noted that the MF subject's motivation with the task could have been higher than that of the LF and HF subjects. It could be argued that this motivation rather than the subject's perceived knowledge systematically influenced the findings of this study and the Bettman and Park (1980) paper, which revealed more extensive processing by MF than by $\mathrm{LF}$ or HF subjects. However, an examination of the category breadth, decision time, and confidence analyses show no evidence of a systematic bias.

Finally, given that there is no commonly accepted (or even proposed) conceptual definition of product familiarity, the results of this study should be interpreted in accordance with how the level of familiarity was operationalized. If, for example, the level of the DM's familiarity is defined differently, e.g., in terms of the amount of knowledge in long-term memory, a different set of research questions may be raised. To illustrate, investigation is needed into the information-acquisition process of decision makers at different familiarity levels. Particular attention needs to be placed on the cognitive processes that are initiated during exposure to communication stimuli and to the content and organization of the information acquired after exposure (Edell and Mitchell 1978; Mitchell 1980). Decision makers at different familiarity levels may reveal differences in encoding strategies for which category breadth is only a part.
The decision makers may also differ in their abilities (and modes) to retrieve this information at a later point in time.

$$
\text { [Received January 1980. Revised April 1981.] }
$$

\section{REFERENCES}

Bennett, Peter D., and Harrell, Gilbert D. (1975), “The Role of Confidence in Understanding and Predicting Buyers' Attitudes and Purchase Intentions," Journal of Consumer Research, 2, 110-7.

Bettman, James R. (1979), An Information Processing Theory of Consumer Choice, Reading, MA: Addison-Wesley Publishing Co.

- and Park, C. Whan (1980), "Effects of Prior Knowledge and Experience on Consumer Decision Processes: A Protocol Analysis," Journal of Consumer Research, 7, 234-48.

Bruner, Jerome S. (1957), "On Perceptual Readiness,"' Psychological Review, 64, 123-52.

— ment Change,' Journal of Abnormal and Social Psychology, $62,231-41$.

Clayton, M. A., and Jackson, D. N. (1961), “Equivalence Range, Acquiescence and Overgeneralization," Educational and Psychological Measurement, 21, 371-82.

Edell, Julie A., and Mitchell, Andrew A. (1978), “An Information Processing Approach to Cognitive Response," in $R e$ search Frontiers in Marketing: Dialogues and Directions, ed. S. C. Jain, Chicago: American Marketing Association, pp. $178-83$.

Hansen, Flemming (1972), Consumer Choice Behavior: A Cognitive Theory, New York: The Free Press.

Howard, J. A., and Sheth, Jagdish A. (1969), The Theory of Buyer Behavior, New York: John Wiley \& Sons.

Kiesler, Charles A. (1966), "Conflict and Number of Choice Alternatives," Psychological Reports, 18, 603-10.

Lichtenstein, Sarah, and Fishhoff, Baruch (1977), "Do Those Who Know More Also Know More about How Much They Know?' Organizational Behavior and Human Performance, $20,159-83$.

Mitchell, Andrew A. (1980), " Cognitive Process Initiated by Exposure to Advertising," in Information Processing Research in Advertising, ed. Richard Harris, Hillsdale, NJ: Lawrence Erlbaum Associates.

Park, C. Whan (1978), "A Conflict Resolution Choice Model," Journal of Consumer Research, 5, 124-37.

_ Stages of the Familiarity Curve: Promotional Implications," Journal of Advertising, 6, 10-6.

- and Winter, Frederick W. (1979), "Product Quality Judgment: Information Processing Approach," Journal of the Market Research Society, 21, 211-7.

Payne, J. W. (1976), "Task Complexity and Contingent Processing in Decision Making: An Information Search and Protocol Analysis," Organizational Behavior and Human Performance, 16, 366-87.

Pinson, Christian (1978), 'Consumer Cognitive Styles," in Marketing: Neue Ergebnisse aus Forschung und Praxis, ed. E. Topritzhofer, Dusseldorf, Germany: Westdeutscher, Verlag.

Pollay, Richard W. (1970), "A Model of Decision Times in Difficult Decision Situations,' Psychological Review, 77, 274-81. 
Rothschild, Michael L., and Houston, Michael J. (1980), "Individual Differences in Voting Behavior: Further Investigations of Involvement," in Advances in Consumer Research, Vol. 7, ed. Jerry C. Olson, San Francisco: Association for Consumer Research.

Sheth, Jagdish N., and Venkatesan M. (1968), "Risk-Reduction Processes in Repetitive Consumer Behavior," Journal of Marketing Research, 5, 307-10.
Tversky, A., and Kahneman, Daniel (1974), "Judgment and Uncertainty: Heuristics and Biases," Science, 185, 1124-31.

Wright, Peter, and Kriewall, Mary Ann (1980), "State-of-Mind Effect on the Accuracy With Which Utility Functions Predict Marketplace Choice," Journal of Marketing Research, 17 , 277-93.

\section{-WANTED - COPIES OF DECEMBER $1976 J C R$}

The Business Office of the Journal of Consumer Research would like to buy back 50 copies (in good condition) of the December 1976 issue of JCR (Vol. 3, No. $3)$, and will pay $\$ 5.00$ per copy.

Interested persons should contact the Managing Editor immediately by phone or mail. 